\title{
Liver Motion Due to Needle Pressure, Cardiac, and Respiratory Motion During the TIPS Procedure
}

\author{
Vijay Venkatraman ${ }^{2}$, Mark H. Van Horn ${ }^{1}$, Susan Weeks ${ }^{1}$, and Elizabeth Bullitt ${ }^{1}$ \\ ${ }^{1}$ CASILAB, University of North Carolina, Chapel Hill, NC \\ \{mvanhorn, sue_weeks, bullitt\} @med.unc.edu \\ http: / / casilab.med.unc.edu \\ ${ }^{2}$ North Carolina A\&T State University, Greensboro, NC \\ vkvenkat@ieee.org
}

\begin{abstract}
TIPS (Transjugular Intrahepatic Portosystemic Shunt) is an effective treatment for portal hypertension. However, during the procedure, respiration, needle pressure, and possibly other factors cause the liver to move. This complicates the procedure since the portal vein is not visible during needle insertion. We present the results of a study of intraoperative liver motion.
\end{abstract}

\section{Introduction}

This paper characterizes intraoperative hepatic motion caused by respiration and needle pressure during Transjugular Intrahepatic Portosystemic Shunt (TIPS) creation. Endovascular procedures are typically guided by fluoroscopic, projection images. These images generally show only the connected portions of the vasculature "downstream" of the catheter tip. The interventionalist thus cannot simultaneously visualize other important anatomical structures or vascular structures that are either "upstream" or disconnected from the vascular anatomy shown in any particular image.

The goal of TIPS is to relieve portal hypertension in patients with liver failure by creating a permanent connection (shunt) between the portal vein and the hepatic vein. During TIPS, the interventionalist obtains access to the internal jugular vein in the neck, and then selectively catheterizes distally into the hepatic vein. The catheter is exchanged for a needle, which is then advanced through the liver parenchyma into the portal vein. This is considered the most difficult part of the procedure [1]. Up to 10 needle passes may be required to achieve portal vein access [2].

There are numerous difficulties associated with TIPS. For example, during needle advancement, other organs bordering the liver such as the kidney, gallbladder, colon, and stomach as well as the hepatic vein or hepatic artery can be inadvertently punctured [3]. To guide the needle and identify the portal vein, a static image is obtained in one or two image planes after hepatic vein catheterization. This is used to estimate portal vein location for the remainder of the procedure. Needle pressure and respiration can displace both the liver and the attached portal vein by an unknown 
amount. Needle advancement is therefore done "blindly" under conditions in which the portal vein location is unknown and may change during the procedure.

Our group is developing methods of three-dimensional image guidance of the TIPS procedure. The most critical issue is the determination of portal vein position at each moment in time relative to the interventionalist's needle. Two facts ease the problem. First, TIPS patients possess livers that are hard, dense, fibrotic, and generally nondeformable. This means that although both needle pressure and respiration may displace the liver, neither is likely to deform it. A rigid body transformation should therefore be sufficient to indicate change in liver (and thus attached portal vein) position during the procedure. The same transformation can be applied to other portions of the hepatic and portal venous systems relevant to TIPS, which are located within this rigid liver. Second, the liver is bounded on three sides by the bony ribcage and superiorly by the diaphragm. It is additionally tethered by the left and right triangular ligaments and by the falciform ligaments, all of which limit rotation. We therefore assume that liver movement can be described by tracking a single point within the liver, and that the liver can be modeled as a rigid body.

As one of the initial steps in designing a 3D image guidance system, it is desirable to determine the likely range of liver motion during the procedure. Previous analyses of liver motion have examined only the motions likely to occur as a result of respiration. The current report examines displacement of the liver during the TIPS procedure with analysis of the effects of respiration, needle pressure, and possibly heartbeat.

\section{Background}

A comprehensive assessment was reported in a recent paper [4], which surveyed previous reports on hepatic motion due to respiration during percutaneous minimally invasive procedures. The paper analyzed nine previously published studies of respiratory-associated hepatic motion using different imaging modalities (fluoroscopy, scintigraphy, ultrasound, optical tracking, and MRI). This paper reports that all studies agreed that rostro-caudal motion is the most significant and that measurements of movement in both the anterior-posterior (AP) and lateral directions vary markedly with the assessment technique used.

Korin et al. [5] and Davies et al. [6] suggested that clinically significant liver motion could be approximated effectively by rostro-caudal movement (10-38 mm) alone with minimal motion in the other axes of motion $(2.5 \mathrm{~mm})$. Recent studies by Herline et al. [7], Shimizu et al. [8] and Rohlfing et al. [9] suggest that translations along the other axes (anterior-posterior translation: 1-12 $\mathrm{mm}$ and lateral translation: 1$9 \mathrm{~mm}$ ) are significant and cannot be neglected. Based on these considerations, it is clear that lateral and AP translations are significant, particularly when tracking discrete targets within the liver. However, none of these reports examined liver displacement as a result of needle pressure or heartbeat during the TIPS procedure. 


\section{Methods}

Our approach to tracking intraoperative liver motion employs a contrast-filled, ovoid balloon that is inserted into a hepatic vein at the beginning of each procedure. The operation is guided by determining the balloon location in simultaneously acquired AP and lateral fluoroscopic views separated by approximately $90^{\circ}$. Given prior knowledge of the relationship between the two fluoroscopic views, this pair of $2 \mathrm{D}$ points representing the balloon centroid in each projection image can be then reconstructed into 3D. Under the two assumptions that the diseased liver is a predominantly rigid structure and that rotation is limited by the liver's restraining ligaments, this 3D point can then be tracked over time to provide information about liver (and thus portal vein) displacement during the procedure.
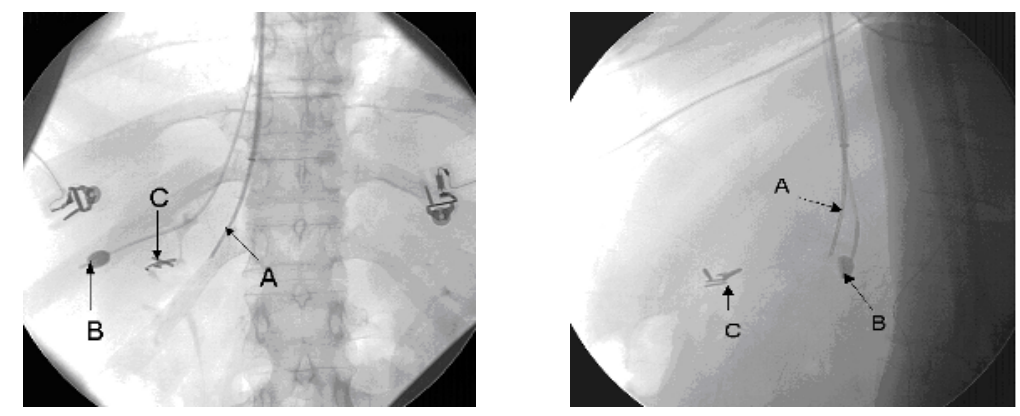

Fig. 1. Typical fluoroscopic images showing the (A) TIPS needle, (B) balloon, and (C) surgical clips. Note that the portal vein is not visible in either image

The current report examines 3D balloon excursion during thirteen image sequences obtained in four subjects undergoing a TIPS procedure. We examined the displacement induced by respiration and needle insertion by using paired image sequences for each study. Image sequences were captured using two synchronized PCs at 15 frames per second with a resolution of 884-by-884 pixels. Each image was time-stamped and stored for post-processing.

Balloon locations within each image were detected automatically on each fluoroscopic image. The algorithm detected the balloon projection by convolving each 2D image with a Laplacian of a Gaussian (LoG) kernel, thresholding the output image, and providing a probability distribution function that includes such features as area, area-to-perimeter ratio, and eccentricity. This probability function was used to identify the correct image feature. The tracking process automatically calculated the pixel $(\mathrm{x}, \mathrm{y})$ coordinates representing the centroid of each balloon projection using a connected component algorithm. Subsequent balloon tracking was performed in similar fashion throughout the time sequence with an ROI of 192-by-192 pixels centered on the previously estimated balloon location, thus allowing real-time tracking of the balloon on each image.

In order to make 3D measurements from the biplane views, projection matrices for each view must be computed. For calibration, we used a Plexiglas phantom containing an array of metallic spheres in known locations, and assumed a pinhole 
camera model. Using the known control point locations and an x-ray image of the phantom, the projection matrices are computed by minimizing the distance between the ideal projections and the observed pixel coordinates of the control points $[10,11]$.

Given each pair of pixel coordinates for the balloon and the projection matrices for each camera system, the $3 \mathrm{D}$ point can be reconstructed by triangulation $[10,11]$. A pair of points in correspondence is considered from the biplane images: $m=(u, v)^{\mathrm{T}}$ and $\mathrm{m}^{\prime}=\left(\mathrm{u}^{\prime}, \mathrm{v}^{\prime}\right)^{\mathrm{T}}$ and reconstructed into a $3 \mathrm{D}$ point $\left(\mathrm{X}=[\mathrm{x}, \mathrm{y}, \mathrm{z}, \mathrm{t}]^{\mathrm{T}}\right)$. For this paper, we report balloon motion in a coordinate system where the $\mathrm{x}$-axis represents motion from the patient's left-to-right (lateral), the y-axis represents motion from caudal-to-cranial, and the z-axis represents motion from back-to-front (anterior-posterior). For each image pair, balloon motion was tracked over time in $\mathrm{x}, \mathrm{y}$, and $\mathrm{z}$; the maximum excursion was also calculated along each axis.

As previously noted, this work has proceeded with the assumptions that the liver (and all structures within it) move rigidly and that rotation is minimal. If these assumptions are correct, point tracking of an intrahepatic balloon may provide a reasonable estimate of the displacement of other structures within the liver, such as the portal vein. We tested these assumptions in one TIPS patient who had surgical clips within the liver, where the relative motion between these clips and the tracking balloon was used to check the validity of the assumptions. Two paired image sequences were used. In the first sequence, only respiration was involved. In the second, images were acquired during needle insertion.

\section{Results}

The graphs below show typical movement of the liver in the three independent axes due to respiration and needle push in a typical subject studied.
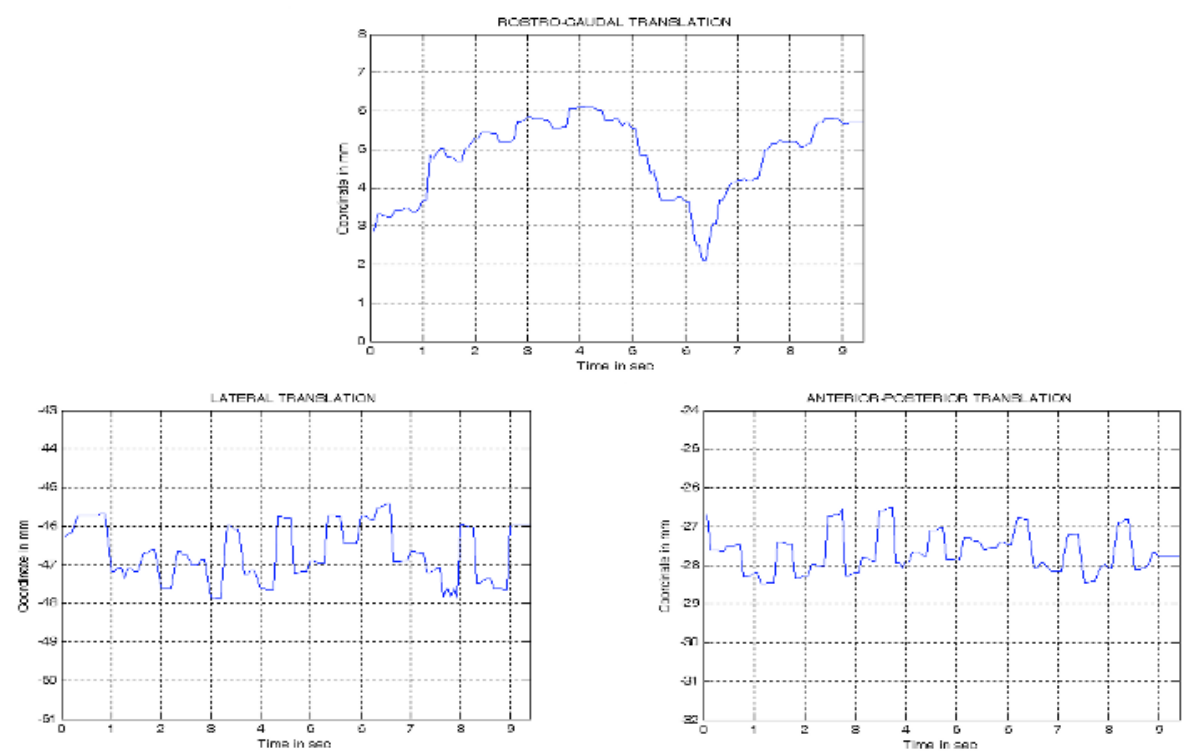

Fig. 2. Liver motion during respiration 
Figure 2 shows the hepatic motion due to respiration in the three axes. Note that with respiration the predominant displacement occurs rostro-caudally (Table 1). However, the graph also shows oscillations at $1 \mathrm{~Hz}$, which are consistent with the cardiac cycle. The frequency of the oscillations was verified using power spectral density analysis.

Table 1. Liver Displacement due to respiration

\begin{tabular}{lccc}
\hline & $\begin{array}{c}\text { Displacement } \\
(\mathrm{mm})\end{array}$ & $\begin{array}{c}\text { Mean } \\
(\mathrm{mm})\end{array}$ & $\begin{array}{c}\text { Standard } \\
\text { Deviation }(\mathrm{mm})\end{array}$ \\
\hline Lateral & $1.2-2.5$ & 1.9 & 0.4 \\
Rostro-Caudal & $3.9-12.3$ & 7.3 & 3.0 \\
Anterior-Posterior & $1.9-3.6$ & 2.5 & 0.7 \\
\hline
\end{tabular}
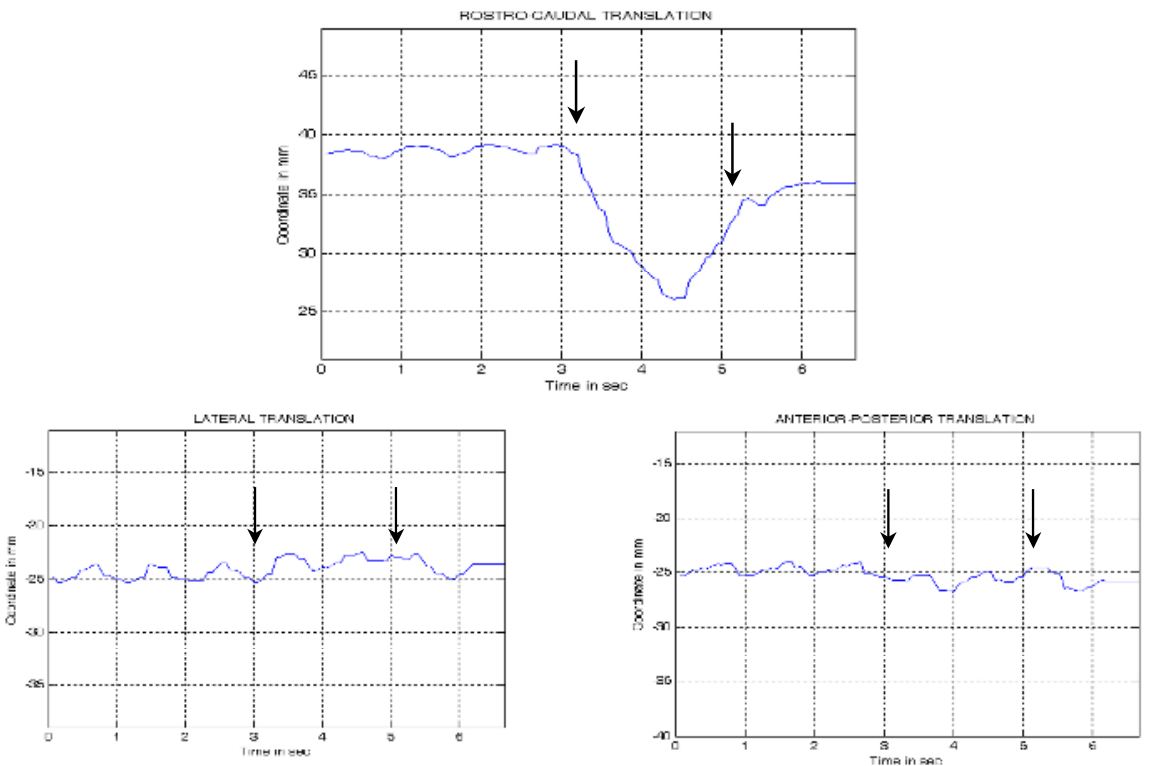

Fig. 3. Liver motion during needle insertion

Figure 3 shows an example of the hepatic motion during the needle push through the liver during the TIPS Procedure. The push started at around 3 seconds and continues for approximately 2 seconds as indicated by the arrows. Again, most of the movement is rostro-caudal, but slight motion also occurs along the other axes. Between patients, the movement during the needle push was highly variable in the rostro-caudal direction (Table.2). The $1 \mathrm{~Hz}$ oscillations were present as previously noted. 
Table 2. Liver displacement during needle insertion

\begin{tabular}{lccc}
\hline & $\begin{array}{c}\text { Displacement } \\
(\mathrm{mm})\end{array}$ & $\begin{array}{c}\text { Mean } \\
(\mathrm{mm})\end{array}$ & $\begin{array}{c}\text { Standard } \\
\text { Deviation }(\mathrm{mm})\end{array}$ \\
\hline Lateral & $1.4-3.1$ & 2.2 & 0.7 \\
Rostro-Caudal & $2.7-13.2$ & 7.4 & 3.9 \\
Anterior-Posterior & $1.1-2.8$ & 2.2 & 0.7 \\
\hline
\end{tabular}

From tables 1 and 2, we can deduce that the rostro-caudal translation is the predominant motion but the anterior-posterior and lateral translations may produce several millimeters displacement. The magnitude of the displacement varies from patient to patient.

To validate our assumptions regarding the rigidity of the liver and modeling liver motion with translation only, we analyzed the relative $3 \mathrm{D}$ positions of the tracking balloon and a set of surgical clips in one patient. These results are summarized in table 3. While there is a slight variation, the surgical clips and the balloon move in similar directions indicating that our assumptions are generally valid.

Table 3. Mean/standard deviation of balloon-to-clip measurements in $\mathrm{mm}$

\begin{tabular}{lccc}
\hline Lateral $(\mathrm{mm})$ & $\begin{array}{c}\text { Rostro-Caudal } \\
(\mathrm{mm})\end{array}$ & $\begin{array}{c}\text { Anterior- } \\
\text { Posterior }(\mathrm{mm})\end{array}$ \\
\hline Respiration & $35.7 / 0.3$ & $1.7 / 0.4$ & $37.7 / 0.4$ \\
Needle push & $35.8 / 0.4$ & $1.5 / 0.2$ & $39.2 / 0.2$ \\
\hline
\end{tabular}

These data suggest that the 3D position of a region of interest within the liver such as surgical clips or the portal vein may be estimated to within $2 \mathrm{~mm}$ given the $3 \mathrm{D}$ position of the tracking balloon and initial offset to the region.

\section{Discussion}

This paper studies motion of a point within the liver during the TIPS procedure. Our results are consistent with those of other groups who have studied the effect of respiratory motion on liver displacement, concluding that translation along all the axes are significant with the rostro-caudal translation being the predominant translation.

This paper also reports on liver motion during needle pressure. We are unaware of another group reporting a similar study. We conclude that needle pressure displaces the liver primarily rostro-caudally, but can produce displacement of several millimeters along the other two axes.

An unexpected finding is the approximately $1 \mathrm{~Hz}$ oscillations along all axes. These oscillations are consistent with the cardiac rhythm. Such motion is less than the 
predominantly rostro-caudal motion induced by respiration or needle pressure, but the induced motion can be significant when the radiologist's goal is to reach a target that cannot be seen.

A limitation of our approach, in common with the majority of other studies of liver motion, is that we track motion of only a single point within the liver. Rotational movement and deformations therefore cannot be assessed. However, as discussed earlier, it is reasonable to assume that rigid body transformations may be sufficient when analyzing the rigid, diseased liver in patients undergoing TIPS procedures, and that the liver's attached ligaments are likely to preclude major rotational movement. Support for this hypothesis is provided by our analysis of the motion of implanted surgical clips with respect to the tracking balloon wedged within a hepatic vein. Although further studies are required, the current report suggests that tracking a balloon visualized on intraoperative, biplane fluoroscopic views can be an effective means of tracking structures within the liver, such as the portal vein, during respiration, heartbeat, and needle pressure.

Acknowledgments. This work was supported by R01 HL69808 NIH-HLB.

\section{References}

1. LaBerge JM. Transjugular Intrahepatic Portosystemic Shunts Technique Using the Wallstent Endoprosthesis in Portal Hypertension: Options for Diagnosis and Treatment. Society of Cardiovascular \& Interventional Radiology (1995)

2. Rees Cr, Niblett RL, Lee SP, Diamond NG, Crippin JS. Use of Carbon Dioxide as a Contrast Medium for Transjugular Intrahepatic Portosystemic Procedures. JVIR (1994), 5:383-386

3. LaBerge JM. Hepatic Anatomy Relevant to Transjugular Intrahepatic Portosystemic Shunts in Portal Hypertension: Options for Diagnosis and Treatment. Society of Cardiovascular \& Interventional Radiology (1995)

4. Clifford MA, Banovac F, Levy E, Cleary K. Assessment of Hepatic Motion Secondary to Respiration for Computer Assisted Interventions. Computer Aided Surgery, 7:291-299 (2002)

5. Korin HW, Ehman RL, Riederer SJ, Felmlee JP, Grimm RC. Respiratory Kinematics of the Upper Abdominal Organs: A Quantitative Study. Magn Reson Med (1992), 23:172178

6. Davies SC, Hill AL, Holmes RB, Halliwell M, Jackson PC. Ultrasound Quantitation of Respiratory Organ Motion in the Upper Abdomen. Br J Radiol (1994), 67:1096-1102

7. Herline AJ, Stefansic JD, Debelak JP, Hartmann SL, Pinson CW, Galloway RL, Chapman WC. Image Guided Surgery: Preliminary Feasibility Studies of Frameless Stereotactic Liver Surgery. Arch Surg (1999), 134:644-649; discussion 649-650

8. Shimizu S, Shirato H, Xo B, Kagei K, Nishioka T, Hashimoto S, Tsuchiya K, Aoyama H, Miyasaka K. Three-Dimensional Movement of a Liver Tumor Detected by High-Speed Magnetic Resonance Imaging. Radiother Onco (1999), 50:367-370

9. Rohlfing T, Maurer CR, O'Dell WG, Zhong J. Modeling Liver Motion and Deformation During the Respiratory Cycle Using Intensity-Based Free-Form Registration of Gated MR Images. In: Medical Imaging: Visualization, Display, and Image-Guided Procedures. Proceedings of SPIE, vol 4319. SPIE (2001), 337-348

10. Gang X and Zhengyou Z. Epipolar Geometry in Stereo, Motion and Object Recognition. Kluwer Academic Publishers (1996)

11. Faugeras O. Three-Dimensional Computer Vision: A Geometric Viewpoint. MIT Press (1996) 